Sports Training

\title{
What is the effect of vigorous exercise on the brain?
}

\author{
Daniel Carvalho Pereira ${ }^{1}$ (D) , Fabiano de Souza Fonseca² ${ }^{2}$, , Hassan Mohamed Elsangedy ${ }^{1}$ \\ ${ }^{1}$ Universidade Federal do Rio Grande do Norte, Departamento de Educação Física, Natal, RN, Brasil; \\ ${ }^{2}$ Universidade Federal Rural de Pernambuco, Departamento de Educação Física, Recife, PE, Brasil \\ Associate Editor: Eduardo Lusa Cadore, UFRGS/Porto Alegre, RS, Brasil
}

\begin{abstract}
Aims: This study aimed to verify whether vigorous exercise is capable of generating mental fatigue. Methods: To do so, 16 young adult male $(29.4 \pm 5.2$ years old $)$ cyclists $(5.4 \pm 4.6$ years of training $)$ underwent three visits: 1) control session (rest); 2) session with cognitive demand (20 minutes of AX-CPT); 3) session with vigorous exercise $(10 \mathrm{~km}$ time trial). Mental fatigue was assessed using the visual analog scale of fatigue in the preand post-session moments. A two-way ANOVA of repeated measures followed by the Bonferroni posthoc was used to verify the effect of the condition (control, cognitive demand, and exercise) and time (pre and post) interaction. The paired " $\mathrm{t}$ " test was used to compare the delta of mental fatigue (post - pre) of the sessions. The partial eta squared was used to determine the effect size of the variance. The significance adopted was $p<0.05$. Results: A condition $\mathrm{x}$ time interaction was observed $(\mathrm{F}(2.30)=5.349, \mathrm{p}=0.010$, partial $\eta 2=0.263)$. When comparing the deltas, a mean difference was found between the control and vigorous exercise $(\mathrm{p}=0.033)$ conditions and cognitive demand and vigorous exercise $(\mathrm{p}=0.017)$ conditions. Control and cognitive demand sessions showed no difference $(\mathrm{p}=$ 0.801). Conclusion: The findings suggest that intense physical exercise seems to cause mental fatigue after practice.
\end{abstract}

Keyword: vigorous; exercise; brains; acute effects

\section{Introduction}

Several factors interfere with physical performance such as cardiorespiratory, neuromuscular, technical/tactical, and cognitive skills $s^{1,2}$ this comparative study sought to determine whether professional road cyclists have superior inhibitory control and resistance to mental fatigue compared to recreational road cyclists. nnMethods After preliminary testing and familiarization, eleven professional and nine recreational road cyclists visited the lab on two occasions to complete a modified incongruent colour-word Stroop task (a cognitive task requiring inhibitory control. All of these variables can suffer losses from the breakdown of the body's homeostasis due to physical exercise, and a known state of fatigue can emerge, the vigorous-intensity, causes greater physiological demand when compared to light and moderate exercise, thus it can impose fatigue of greater magnitude Some theories discuss fatigue models in the sports context ${ }^{3}$ and the musculoskeletal system. Moreover, in many sports (for example, running and cycling, as a section of the literature recently explored mental fatigue as a possible predictor of physical performance in sports of endurance ${ }^{4}$.

Mental fatigue is conceptualized as a psychobiological state characterized by feelings of tiredness and lack of energy caused by long periods of cognitive demand ${ }^{4,5}$ we examined whether error-related brain activity, indexing performance monitoring by the anterior cingulate cortex (ACC. In team sports, it leads to slower running speed ${ }^{6}$, shorter distance, worse speed, lower passing accuracy, ball control ${ }^{7}$, and decision-making errors ${ }^{8} 15$-min Stroop task, and 30-min Stroop task. Inhibitory control was accessed by the Stroop task (accuracy and response time. In cycling, a previous study showed that mental fatigue causes increased perception of effort, impairing performance without changing traditional physiological markers such as lactate and heart rate ${ }^{4}$. A possible mechanism which explains this effect in the exercise was proposed by Martin ${ }^{9}$, in which he suggests that this occurs through the dopamine/adenosine balance in which the presence of adenosine makes it impossible to use dopamine in the brain, dopamine is also linked to cognition ${ }^{10}$, this balance, in turn, may also causing inhibition difficulties and worsening working memory ${ }^{11}$.

In order to understand the impact of this condition on performance, instruments which require cognitive demands through sustained attention, inhibition of response, and goal maintenance have been used, the most common instruments are computer tests the Stroop task, ${ }^{7}$ and the $\mathrm{AX} \mathrm{CPT}^{4}$. In addition, other common situations such as using smartphones before exercising can also cause this condition ${ }^{12}$. However, the literature normally investigates cognitive stimulation prior to exercise ${ }^{12,13}$, disregarding the effects of physical exercise' on mental fatigue. Some studies have recently shown that physical exercise can cause mental fatigue; for example, $\mathrm{Xu}^{15}$ performed an experimental protocol on a cycle ergometer ${ }^{15}$. Some studies with team sports have also 
identified mental fatigue, for example, players pointed out mental fatigue after a complete rugby match ${ }^{16}$, while a netball match caused similar mental fatigue to 35 minutes of involvement in the Stroop task in high-level athletes ${ }^{17}$, corroborating that exercise capacity causes mental fatigue.

Assessing the impact of physical exercise on mental fatigue using team sports modalities may present bias due to intervening factors because it is an open modality with interminable efforts, it can cause different cognitive demands (e.g., decision-making, attention, anticipation $)^{18}$. On the other hand, the time trial cycling test can provide more reliable results considering in which the context is relatively stable and controlled in relation to executive functions and cognitive demands with a smaller number of variables to be controlled (i.e. pace control, concentration in the course) ${ }^{19}$. Thus, identifying the impact of vigorous exercise in this regard can help to create strategies for athletes and coaches in order to minimize the harmful effects of this condition on performance.

In view of the above, we hypothesize that vigorous physical exercise will cause mental fatigue. Given this, the objective of this study was to verify the effect of vigorous exercise on mental fatigue.

\section{Methods}

\section{Study Design}

This is an experimental, randomized cross-over study. Each participant performed the evaluation procedures in 3 visits: i) a control session which consisted of anamnesis, health evaluation (Physical Activity Readiness Questionnaire (PAR-Q), familiarization with the visual analog scale and collection of the perception of mental fatigue at rest; ii) session with cognitive demand; and iii) a session with vigorous exercise, volunteers performed all sessions at the same time as the first session. Thus, we used 3 experimental sessions in order to answer our objective, with 2 sessions being used as control (control session and cognitive demand session), and one exercise session (exercise session) had similar intervention procedures, except for interventions that were specific to each condition. If the participant did not attend one of the sessions or did not answer any protocol of this research, he was excluded from the analysis. The subjects answered the visual analog scale before and after the interventions (rest / AX-CPT / TT10km). The sessions with cognitive demand and intense exercise were randomized and balanced. The minimum interval between each visit was $48 \mathrm{~h}$. The participants were instructed to refrain from using caffeine, tobacco, or alcohol, as well as to not perform moderate and/ or vigorous physical activity 24 hours before each visit.

\section{Participants}

A total of 16 volunteers who participated in the study, considered recreational cyclists, with the characteristics described in Table 1. Figure 1 describes the study flowchart. The eligibility criteria were: (1) Male individuals; (2) age between 18 and 40 years; and (3) travel at least $50 \mathrm{~km}$ per week.

Table 1 - Description of sample characteristics and exercise session $(n=16)$

\begin{tabular}{cc}
\hline Variables & Mean $(\mathbf{C I}$ 95\%) \\
\hline Age (years) & $29.4(26.14 ; 32.15)$ \\
Body fat $(\%)$ & $22.1(17.79 ; 26.40)$ \\
Years of experience & $5.4(2.21 ; 7.62)$ \\
Weekly frequency (days/week) & $3.5(2.93 ; 4.64)$ \\
Weekly volume (km/week) & $123.8(95.52 ; 165.91)$ \\
Maximum power (watts) & $274.2(249.52 ; 307.22)$ \\
\hline
\end{tabular}

Note: $\mathrm{CI}=$ Confidence interval

All participants signed the consent form to participate in the study. The study was approved by the institutional ethics committee (CAAE: 2.444.566/2017).

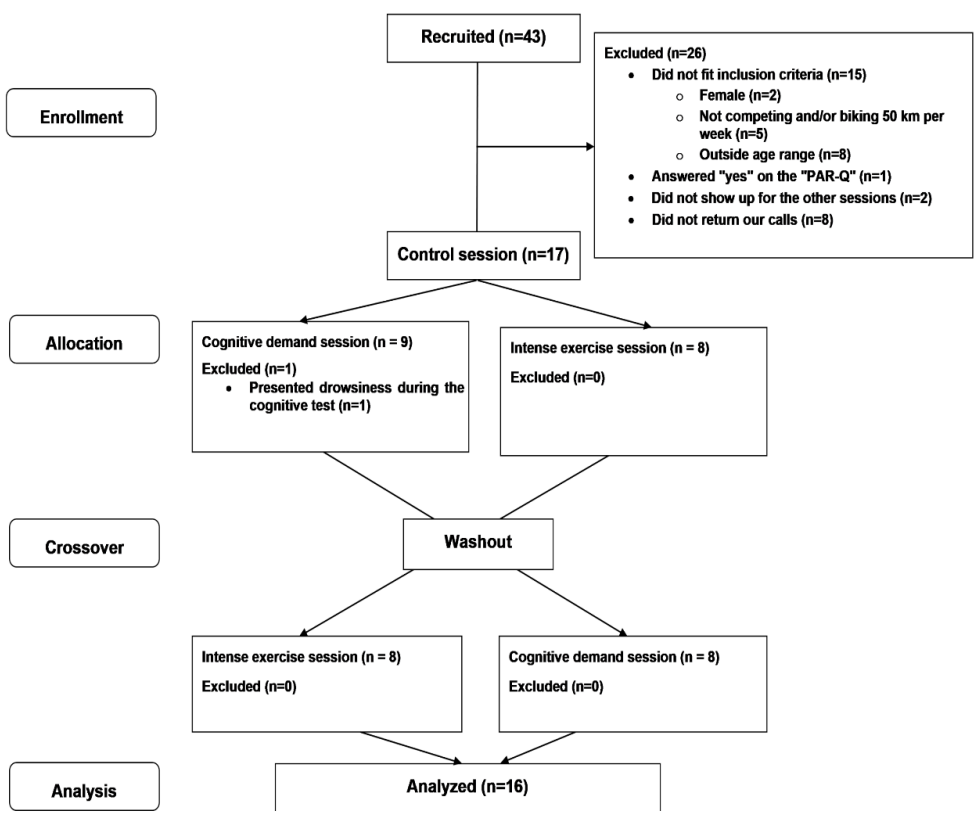

Figure 1 - Study flowchart 


\section{Procedures/Instruments}

\section{Visual Analog Scale (VAS)}

A visual analog scale was used in this study to check mental fatigue (pre and immediately after). The VAS is a one-dimensional scale with eleven points ranging from 0 to 10 . During each session, the participant was instructed to point out a number that represented their mental fatigue at the time, with zero representing no fatigue and ten representing the greatest possible mental fatigue. The numerical descriptors used were: 0-2 "no fatigue", 2-4 "light fatigue", 4-6 "moderate fatigue", 6-8 "strong fatigue", and 8-10 "extremely fatigued"20-23impaired function, and greater disability. It was aimed to explore the effect of the presence of FM on fatigue in patients with PsA comparing with controls. InMethods: Fifty patients with PsA and 34 sex-age matched controls were enrolled. In patients; pain was assessed by Visual Analogue Scale, disease activity by DAS-28, enthesitis by The Leeds Enthesitis Index. Fatigue level of all participants was evaluated by Multidimensional Assessment of Fatigue. In all participants, FM was determined according to 2010 American College of Rheumatology criteria. InResults: Seventeen patients with PsA (34\%. This scale was chosen mainly to facilitate the measurement after the intense exercise

\section{Control Condition}

The participant remained at rest in the supine position for $20 \mathrm{~min}$, and the resting heart rate was checked $\left(\mathrm{HR}_{\mathrm{Rep}}\right)$. The instructions given were to not fall asleep, not communicate, and not move during that time. The lowest HR value was assumed to be $\mathrm{HR}_{\text {Rep }}$. This time was determined due to the same duration of the cognitive control test.

\section{Cognitive Demand Condition}

The cognitive demand was caused by the AX-Continuous Performance Task (AX-CPT) for $20 \mathrm{~min}$. It is an attentional test capable of increasing cognitive demand ${ }^{4}$. The inclusion of this cognitive task served as a control for mental fatigue, considering that it seems to occur 30 minutes into participating in this task, while 20 minutes of this test would increase the cognitive load, however it would not cause mental fatigue ${ }^{13}$.

In the AX-CPT, each stimulus stays in the center of the screen for $250 \mathrm{~ms}$, with an interval of $1000 \mathrm{~ms}$ in between each one (Figure 2). Participants were instructed to press "2" on the keyboard whenever a letter showed up, except when the letter " $X$ " was preceded by the letter " $A$ ". In this case, participants were told to press " 3 ". Any other response was considered an error ${ }^{24}$. This test consists of 120 stimuli in the following proportion: $70 \% \mathrm{~A}-\mathrm{X}$ (84 tasks), 10\% B-X (12 tasks), $10 \%$ A-Y (12 tasks), and 10\% B-Y (12 tasks). We used the E-prime v.1.2 software (Psychological Software Tools Inc) program for the test.

\section{Vigorous Exercise Condition}

A $10 \mathrm{~km}$ time trial protocol (TT10km) was used on the cyclosimulator). Before starting, the subjects were free to adjust the height and distance of the ergometer saddle and handlebars (Velotron ${ }^{\text {TM }}$ - RacerMate ${ }^{\circledR}$, Seattle, WA, USA. Pre-calibrated) The test previously included five minutes of warm-up with load and self-selected cadence. Participants were instructed to cover the $10 \mathrm{~km}$ in the shortest possible time, being allowed to control power (watts) and cadence throughout the session, they were also aware of the distance traveled. All participants received verbal incentives each $2 \mathrm{~km}$, in a standard way in order to perform the test with the best physical performance (i.e let's go; i.e. keep the pace; i.e.speeds up).

The reserve heart rate was used as an intensity marker $\left(\mathrm{HR}_{\mathrm{Res}}\right)$, is calculated from the formula: $\mathrm{HR}_{\mathrm{Res}}=\mathrm{HR}_{\mathrm{Max}}-\mathrm{HR}_{\mathrm{Rep}}{ }^{25}$. The reserve heart rate intensity percentage was verified through the mean of the HR during the TT10km using the formula $\left(\mathrm{HR}_{\text {Mean }}\right.$ $\left.-\mathrm{HR}_{\mathrm{Rep}}\right) /\left(\mathrm{HR}_{\mathrm{Res}} \times 100\right)^{26,27}$. In addition, to ensure vigorous execution, $\%$ HR and power was monitored throughout the course, if there was a marked decrease in these variables, the subject was oriented to increase the effort. A cardiac monitor RS800CX, Polar ${ }^{\circledR}$, Finlândia, was used in all sessions.

\section{Statistical analysis}

The data normality was verified by the Shapiro-Wilk test, and asymmetry and kurtosis were used to test the normal distribution. The results are expressed as mean and standard deviation according to a $95 \%$ confidence interval. A Two-way ANOVA of repeated measures accompanied by the post hoc Bonferroni test was performed to verify the effect of condition $\mathrm{x}$ time interaction of VAS. The partial eta squared (partial $\eta^{2}$ ) was used to verify the variance effect size of ANOVA of repeated measures. The normality of the residuals was verified by the normal Q-Q graph. The paired t-test was used to compare the delta of mental fatigue (post - pre) of the sessions. The data were analyzed using the SPSS ${ }^{\circledR}$ version 20.0 program (IBM, Inc., Chicago, USA). An alpha level of $p<0.05$ was used to determine statistical significance.

\section{Results}

The statistical power calculation was carried out a posteriori. Differences between VAS were conducted to determine the attained power based on the size of the investigated sample, an alpha of $5 \%$, and the obtained effect size. The obtained power for the group effect analysis was $85 \%$ (3 conditions, 2 times). The TT10km lasted on average \pm SD $20.28 \pm 3.1$ minutes, with medium intensity $\pm \mathrm{SD}$ of $84.5 \pm 12.36 \%$ of Heart rate reserve.

When comparing the three conditions (control, cognitive demand and exercise) in two moments (pre and post), the Twoway Anova identified interaction condition * time $(\mathrm{F}(2.30)=$ 5.349, $\mathrm{p}=0.010$, partial $\left.\eta^{2}=0.263\right)$, as well as a difference in time $\left(\mathrm{F}(1.15)=21.793, \mathrm{p}=0.000\right.$, partial $\left.\eta^{2}=0.592\right)$. No 
difference was found between the conditions $(\mathrm{F}(2.30)=1.989$, $\mathrm{p}=0.154$, partial $\left.\eta^{2}=0.117\right)($ Figure A).

The post hoc test revealed an average difference between the times, with increased mental fatigue in the three conditions, control $(0.813 \pm 0.256 ; p=0.006)$, cognitive demand $(0.750 \pm 0.250 ; p=$ $0.009)$ and exercise $(1.775 \pm 0.407 ; \mathrm{p}=0.001)$. When comparing conditions, the only difference identified was in the time between cognitive demand and exercise conditions $(1,000 \pm 0.329 ; p=0.025)$. The other conditions showed no significant difference $(\mathrm{p}>0.05)$.
When analyzing the delta of the conditions (post - pre), an average difference between the control and exercise conditions ( $p=0.033$ ) was found to be $0.96 \pm 1.63$ with a standard error of the average of 0.40 and confidence intervals of $95 \%$ ( 0.09 to 1.83). In addition, a mean difference in the cognitive demand and exercise conditions $(\mathrm{p}=0.017)$ of $1.02 \pm 1.51$ was observed with a standard error of the mean of 0.38 and $95 \%$ confidence intervals ( 0.21 to 1.83$)$. Control and cognitive demand sessions showed no difference $(\mathrm{p}=0.801)$ (Figure $\mathrm{B})$.
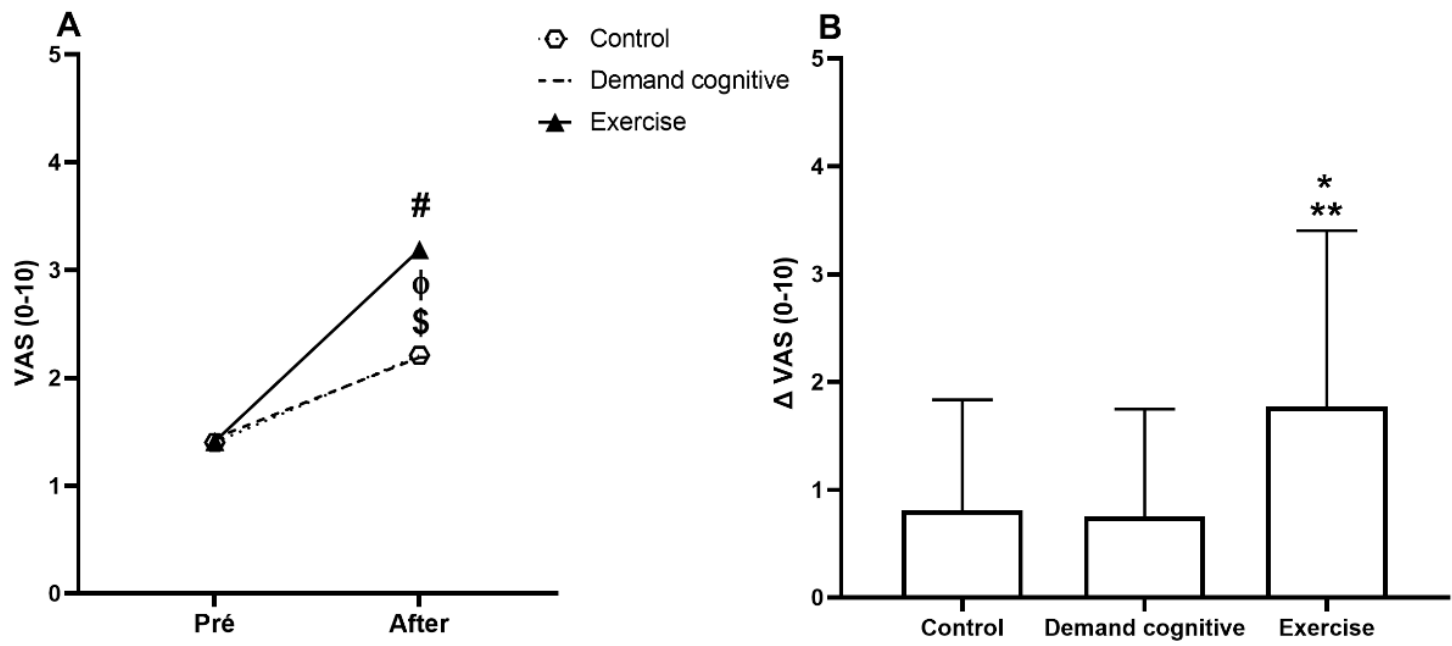

Figure 2 - (A) Behavior of mental fatigue (pre and post-control sessions, cognitive demand, and exercise). Note: (Ф) and (\$) p $<0.05$ difference from the pre and post moments in the control and cognitive demand sessions, respectively. (\#) $\mathrm{p}<0.05$ compared to the pre-exercise and post-session cognitive demand. VAS $=$ Visual Analog Scale. (B) Delta of mental fatigue. Note $\left(^{*}\right) \mathrm{p}<0.05$ compared to the control; (**) $\mathrm{p}<0.05$ compared to cognitive demand. VAS $=$ Visual Analog Scale

\section{Discussion}

This study aimed to verify the effect of cognitive demand and exercise intensity on mental fatigue. The study hypothesis was confirmed, as intense physical exercise caused a mild mental fatigue condition. Our main result was greater mental fatigue in the post-exercise moment, as well as a greater variation pointed out by the greater delta in the exercise session when compared to control and cognitive demand, while the control and cognitive demand conditions did not differ. In this sense, our findings suggest that vigorous endurance exercise can negatively impact the brains causing mental fatigue in its practitioners at the end and after vigorous exercise, so everything that the mental fatigue condition causes can be associated with a post-exercise condition (i.e. difficulty with inhibition and concentration, or low motivation $)^{9}$.

The results of the present study are in agreement with the findings of $\mathrm{Xu}^{15}$, who verified the effect of different exercise protocols performed on a cycle ergometer on brain waves (electroencephalography), identifying that the exercise generated an increase in the beta band and the alpha/beta band ratio linked to mental fatigue. These findings were also similar to those found in studies that used subjective instruments to measure mental fatigue. In the studies by Russel et al. ${ }^{17}$ and Maschiko ${ }^{16}$, higher levels of mental fatigue were identified after 60 minutes of participation in a netball match and after a complete rugby match, respectively (VAS and POMS). Considering these results, it is important to emphasize that although cyclical endurance sports and team sport modalities present differences in physical stimuli (e.g., motor coordination, balance, speed, cardiorespiratory fitness) and cognitive stimuli (e.g., decision-making, attention, anticipation $)^{18}$, the effects on the process of developing mental fatigue were similar.

This mental condition at the end of exercises can be common in several modalities with similar physiological demands and intensity ${ }^{28,29}$ midfielders, forwards and wingabcks. A possible explanation for these results can be supported by the theory of the central governor ${ }^{30}$ by which fatigue is caused, mainly in aerobic exercises, being signaled by the brain acting to preserve the body's cellular homeostasis ${ }^{31,32}$. Trying to add to the pre-existing knowledge, we suggest that the specifics of mental fatigue (i.e. energy synthesis, cortical activation) occur in exercise as 
the brain's first signal to preserve the human body. Therefore, intense exercise possibly causes a change in the dopamine/ adenosine ratio in the brain ${ }^{9}$.

A possible physiological explanation of how this mechanism works may be due to the lactate/dopamine/adenosine ratio ${ }^{9,33,34}$. Aerobic exercise causes an increase in blood lactate, which in turn can increase the production and concentration of extracellular dopamine ${ }^{34}$, meaning that when the lactate concentration increases, the dopamine concentration also increases. This consequently requires the use of $\mathrm{D} 1$ receptors which results in adenosine production during exercise ${ }^{33}$. It is possible that the exposure time to exercise increases adenosine production, decreasing dopamine synthesis in the brain, and consequently triggering mental fatigue ${ }^{9}$. Thus, the appearance of mental fatigue in vigorous-intensity efforts has been observed ${ }^{17,35}$. Caffeine and carbohydrates prove to be effective tools to alleviate this condition, improving physical performance and alleviating mental fatigue $^{36,37}$, possibly due to the caffeine cell structure which inhibits adenosine ${ }^{38}$. We emphasize the difficulty of obtaining direct measures of these mechanisms, and also the need to develop technologies that enable understanding this phenomenon.

Therefore, our study identified intense exercise as mentally stressful. In doing so, the implications associated with this condition such as the increased perception of effort and worsening performance in this condition must be assumed ${ }^{4}$. Furthermore, strategies that can help avoid this condition or minimize its effect, especially at the end of a race, must be used to maintain the athlete's physical performance. In addition to controlling the pre-exercise cognitive load (i.e. using smartphones) which can accelerate the condition of mental fatigue, the administration of caffeine or carbohydrate from the end of the endurance test is also suggested to mitigate the harmful effects of this condition.

A limitation in this study was the low/moderate level of physical conditioning of the sample, considering that highly physically conditioned adults have greater resistance to metal fatigue ${ }^{1}$ this comparative study sought to determine whether professional road cyclists have superior inhibitory control and resistance to mental fatigue compared to recreational road cyclists.। nMethods After preliminary testing and familiarization, eleven professional and nine recreational road cyclists visited the lab on two occasions to complete a modified incongruent colour-word Stroop task (a cognitive task requiring inhibitory control, which can be a variable that interferes with the result. Understanding fatigue and physical exercise as a complex phenomenon but with similar physiological demands in several modalities, especially in sports with high cardiorespiratory demand is necessary, and therefore further studies are indicated with tools which can help in identifying mental fatigue (i.e. EEG) and constitutes an area which can enable optimizing performance in various sports (e.g., running, football, rugby, and basketball).

\section{Conclusion}

Intense physical exercise impacts the mental fatigue of cyclists. Coaches must be attentive and provide strategies that mitigate the harms of this condition, aiming toward maintaining physical performance in an endurance race considering the detrimental effect of this condition on performance.

\section{References}

1. Martin K, Staiano W, Menaspà P, Hennessey T, Marcora S, Keegan $\mathrm{R}$, et al. Superior Inhibitory Control and Resistance to Mental Fatigue in Professional Road Cyclists. Piacentini MF, organizador. PLOS ONE. 2016;11(7):e0159907.

2. Pauw KD, Roelands B, Cheung SS, de Geus B, Rietjens G, Meeusen R. Guidelines to Classify Subject Groups in SportScience Research. Int J Sports Physiol Perform. 2013;8(2):111-22.

3. Chuckravanen D, Bulut S, Kürklü GB, Yapali G. Review of exercise-induced physiological control models to explain the development of fatigue to improve sports performance and future trend. Sci Sports. 2019;34(3):131-40.

4. Marcora SM, Staiano W, Manning V. Mental fatigue impairs physical performance in humans. J Appl Physiol. 2009;106(3):857-64.

5. Lorist MM, Boksem MAS, Ridderinkhof KR. Impaired cognitive control and reduced cingulate activity during mental fatigue. Cogn Brain Res. 2005;24(2):199-205.

6. Smith MR, Marcora SM, Coutts AJ. Mental Fatigue Impairs Intermittent Running Performance: Med Sci Sports Exerc. 2015;47(8):1682-90.

7. Smith MR, Coutts AJ, Merlini M, Deprez D, Lenoir M, Marcora SM. Mental Fatigue Impairs Soccer-Specific Physical and Technical Performance: Med Sci Sports Exerc. 2016;48(2):267-76.

8. Gantois P, Caputo Ferreira ME, Lima-Junior D de, Nakamura FY, Batista GR, Fonseca FS, et al. Effects of mental fatigue on passing decision-making performance in professional soccer athletes. Eur J Sports Sci. 2019;1-21.

9. Martin K, Meeusen R, Thompson KG, Keegan R, Rattray B. Mental Fatigue Impairs Endurance Performance: A Physiological Explanation. Sports Med. 2018;48(9):2041-2051.

10. Westbrook A, Braver TS. Dopamine Does Double Duty in Motivating Cognitive Effort. Neuron. 2016;89(4):695-710.

11. Porkka-Heiskanen T, Kalinchuk AV. Adenosine, energy metabolism, and sleep homeostasis. Sleep Med Rev. 2011;15(2):123-35.

12. Fortes LS, Lima-Júnior D de, Gantois P, Nascimento-Júnior JRA, Fonseca FS. Smartphone Use Among High-Level Swimmers Is Associated With Mental Fatigue and Slower 100- and 200- but Not 50-Meter Freestyle Racing. Percept Mot Skills. 2020;003151252095291.

13. Van Cutsem J, Marcora S, De Pauw K, Bailey S, Meeusen R, Roelands B. The Effects of Mental Fatigue on Physical Performance: A Systematic Review. Sports Med. 2017;47(8):1569-88.

14. Dworak M, Diel P, Voss S, Hollmann W, Strüder HK. Intense exercise increases adenosine concentrations in the rat brain: Implications for a homeostatic sleep drive. Neuroscience. 2007;150(4):789-795.

15. Xu R, Zhang C, He F, Zhao X, Qi H, Zhou P, et al. How Physical Activities Affect Mental Fatigue Based on EEG Energy, Connectivity, and Complexity. Front Neurol. 2018; 9:915.

16. Mashiko T. Position related analysis of the appearance of and relationship between post-match physical and mental fatigue in university rugby football players. Br J Sports Med. 2004;38(5):617-21. 
17. Russell S, Jenkins D, Halson S, Kelly V. Changes in subjective mental and physical fatigue during netball games in elite development athletes. J Sci Med Sport. 2019;S1440244019313581.

18. Casanova F, Oliveira J, Williams M, Garganta J. Expertise and perceptual-cognitive performance in soccer: a review. 2009;8.

19. Brick N, MacIntyre T, Campbell M. Metacognitive processes in the self-regulation of performance in elite endurance runners. Psychol Sport Exerc. 2015;19:1-9.

20. Ulus Y, Akyol Y, Bilgici A, Kuru O. The impact of the presence of fibromyalgia on fatigue in patients with psoriatic arthritis: comparison with controls. Adv Rheumatol. 2020;60(1):1.

21. Abbasi AM, Motamedzade M, Aliabadi M, Golmohammadi R, Tapak L. Study of the physiological and mental health effects caused by exposure to low-frequency noise in a simulated control room. Build Acoust. 2018;25(3):233-48.

22. Laborda A, Medrano J, de Blas I, Urtiaga I, Carnevale FC, de Gregorio MA. Endovascular Treatment of Pelvic Congestion Syndrome: Visual Analog Scale (VAS) Long-Term Follow-up Clinical Evaluation in 202 Patients. Cardiovasc Intervent Radiol. 2013;36(4):1006-14.

23. Gudbjörnsson B, Broman JE, Hetta J, Hällgren R. SLEEP DISTURBANCES IN PATIENTS WITH PRIMARY SJÖGREN'S SYNDROME. Rheumatology. 1993;32(12):1072-6.

24. Cohen JD, Barch DM, Carter C, Servan-Schreiber D. Contextprocessing deficits in schizophrenia: Converging evidence from three theoretically motivated cognitive tasks. J Abnorm Psychol. 1999;108(1):120-33.

25. ACSM. ACSM Guidelines for Exercise Testing and Prescription 10th (1).pdf. Philadelphia Wolters Kluwer; 2018.

26. Garber CE, Blissmer B, Deschenes MR, Franklin BA, Lamonte MJ, Lee I-M, et al. Quantity and Quality of Exercise for Developing and Maintaining Cardiorespiratory, Musculoskeletal, and Neuromotor Fitness in Apparently Healthy Adults: Guidance for Prescribing Exercise. Med Sci Sports Exerc. 2011;43(7):1334-59.

27. Guimarães GC, Farinatti PTV, Midgley AW, Vasconcellos F, Vigário P, Cunha FA. Relationship Between Percentages of Heart Rate Reserve and Oxygen Uptake Reserve During Cycling and Running: A Validation Study. J Strength Cond Res. 2019;33(7):1954-62.

28. Coelho DB, Paixão RC da, Oliveira EC de, Becker LK, Ferreira-Júnior JB, Coelho LG, et al. Exercise intensity during official soccer matches. Braz J Kinanthropometry Hum Perform. 2016;18(6):621.

29. Basso JC, Suzuki WA. The Effects of Acute Exercise on Mood, Cognition, Neurophysiology, and Neurochemical Pathways: A Review. Brain Plast. 2017;2(2):127-52.

30. Noakes TD. The Central Governor Model of Exercise Regulation Applied to the Marathon: Sports Med. 2007;37(4):374-7.
31. Lambert EV. Complex systems model of fatigue: integrative homoeostatic control of peripheral physiological systems during exercise in humans. Br J Sports Med. 2005;39(1):52-62.

32. Noakes TD. Fatigue is a Brain-Derived Emotion that Regulates the Exercise Behavior to Ensure the Protection of Whole Body Homeostasis. Front Physiol [Internet]. 2012 [citado 31 de março de 2020];3. Disponível em: http://journal.frontiersin.org/ article/10.3389/fphys.2012.00082/abstract

33. Avila-Luna A, Gálvez-Rosas A, Durand-Rivera A, RamosLanguren L-E, Ríos C, Arias-Montaño J-A, et al. Dopamine D1 receptor activation maintains motor coordination and balance in rats. Metab Brain Dis. 2018;33(1):99-105.

34. Remblier C, Pontcharraud R, Tallineau C, Piriou A, Huguet F. Lactic acid-induced increase of extracellular dopamine measured by microdialysis in rat striatum: evidence for glutamatergic and oxidative mechanisms. Brain Res. 1999;837(1-2):22-8.

35. Mashiko T, Umeda T, Nakaji S, Sugawara K. Position related analysis of the appearance of and relationship between post-match physical and mental fatigue in university rugby football players. Br J Sports Med. 2004;38(5):617-21.

36. Shabir A, Hooton A, Spencer G, Storey M, Ensor O, Sandford $\mathrm{L}$, et al. The Influence of Caffeine Expectancies on Simulated Soccer Performance in Recreational Individuals. Nutrients. 2019;11(10):2289.

37. Azevedo R, Silva-Cavalcante MD, Gualano B, Lima-Silva AE, Bertuzzi R. Effects of caffeine ingestion on endurance performance in mentally fatigued individuals. Eur J Appl Physiol. 2016;116(11-12):2293-303.

38. Meeusen R, Decroix L. Nutritional Supplements and the Brain. Int J Sport Nutr Exerc Metab. 2018;28(2):200-11.

\section{Corresponding author:}

Daniel Carvalho Pereira

Federal University of Rio Grande do Norte, Avenida Senador Salgado Filho, 3000 Djalma Marinho Community Center - Lagoa Nova, Natal, RN, Brazil. Telephone: +55 (54) 999489943 .

Email: danielcarvalhopereira@gmail.com

Manuscript received on August 25, 2020

Manuscript accepted on October 19, 2020

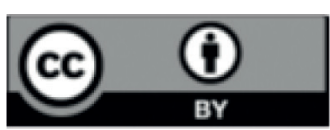

Motriz. The Journal of Physical Education. UNESP. Rio Claro, SP, Brazil - eISSN: 1980-6574 - under a license Creative Commons - Version 4.0 